\title{
Condensed matter physics of biomolecule systems in a differential geometric
} framework

Bohr, Henrik; Ipsen, J. H.; Markvorsen, Steen

Published in:

International Journal of Modern Physics B

Link to article, DOI:

$10.1142 / S 0217979207043828$

Publication date:

2007

Link back to DTU Orbit

Citation (APA):

Bohr, H., Ipsen, J. H., \& Markvorsen, S. (2007). Condensed matter physics of biomolecule systems in a differential geometric framework. International Journal of Modern Physics B, 21 (13 \& 14), 2475-2492. https://doi.org/10.1142/S0217979207043828

\section{General rights}

Copyright and moral rights for the publications made accessible in the public portal are retained by the authors and/or other copyright owners and it is a condition of accessing publications that users recognise and abide by the legal requirements associated with these rights.

- Users may download and print one copy of any publication from the public portal for the purpose of private study or research.

- You may not further distribute the material or use it for any profit-making activity or commercial gain

- You may freely distribute the URL identifying the publication in the public portal

If you believe that this document breaches copyright please contact us providing details, and we will remove access to the work immediately and investigate your claim. 


\title{
CONDENSED MATTER PHYSICS OF BIOMOLECULE SYSTEMS IN A DIFFERENTIAL GEOMETRIC FRAMEWORK
}

\author{
Henrik Bohr \\ Department of Physics, Quantum Protein Centre \\ The Technical University of Denmark, Building 309 \\ DK-2800 Lyngby, Denmark \\ John H. Ipsen \\ Memphys, Department of physics \\ South Danish University, SDU \\ Campusvej 55, DK-5230, Odense M, Denmark \\ Steen Markvorsen \\ Department of Mathematics \\ The Technical University of Denmark, Building 303S \\ DK-2800 Lyngby, Denmark
}

\begin{abstract}
In this contribution biomolecular systems are analyzed in a framework of differential geometry in order to derive important condensed matter physics information. In the first section lipid bi-layer membranes are examined with respect to statistical properties and topology, e.g. a relation between vesicle formation and the proliferation of genus number. In the second section differential geometric methods are used for analyzing the surface structure of proteins and thereby understanding catalytic properties of larger proteins.
\end{abstract}

\section{SECTION I: Thermodynamics and topology of closed membranes}

In this section we shall describe the lipid bilayer membrane system in differential geometrical terms by a curvature elasticity Hamiltonian (Canham-Helfrich) and analyse it especially concerning it's topological features. Phase transitions can be studied when varying the coefficients $\kappa, \bar{\kappa}$ representing respectively the bending rigidity and the Gaussian curvature. It is found that the Hamiltonian has no lower bounds for $\bar{\kappa}$ positive, indicating that handles and tunnels will be formed in large numbers spontaneously in the phase region of $(\kappa, \bar{\kappa} \geq 0)$. For $\bar{\kappa} \leq 0$ apparently there are two phases where either small vesicles are formed in large numbers or large, single vesicles appear, with a second order phase transition in between.

\section{Introduction}

Aggregates formed by small amphiphillic molecules in water display a remarkable structural richness, e.g. micellar, hexagonal and bilayer structures [??]. Furthermore monolayer structures can be formed in water-air or water-hydrocarbon interfaces. Amphiphiles constitute a very extensive class of compounds, which count common substances like soaps, alcohols and lipids. The lipid bilayer structures are of particular interest because they play an essential role in the organization of biological cells. Hydrated lipid bilayer systems display a wealth of polymorphic transitions, however their possible significance in biological membranes are still unrevealed. Although the discussion in this section is restricted to lipid bilayer structures it may be applicable to a range of other amphiphillic surfactant systems. 
Theory has been of limited help in the characterization at the large-scale structural transition properties of lipid bi-layers in excess water. The stabilisation of the lipids in a bilayer structure is understood in the framework of a thermodynamics theory describing the interplay between molecular interaction free energy, molecular geometry and entropy [??]. This analysis has been supplemented by thermodynamics considerations based on electrolyte double-layer theory which can give a description of the stability of simple bilayer shapes like cylinders and spheres.

A popular phenomenological theory for the description of shapes of individual lipid bi-layers is the Canham-Helfrich model [ ??]. This model has even proven to be successfull in the description of shape transformations of biological membranes like erythocytes [??]. Furthermore this model has served as the basis for recent studies on the effects of thermal undulations

undulations?

on the forces between bi-layers [??] and the possibility of order-disorder transitions in macroscopic conformations of membranes [ ??, ??, ??]. However, a full statistical mechanical treatment of this model is not possible before the partition function over all shapes and topologies can be handled analytically or numerically. This is the issue the present paper is concerned with.

In this contribution we demonstrate that it is possible, within a mean-field approach, to obtain important information about the phase behaviour of the theory when only closed membranes are considered. The information is concerned with the membrane stability against changes in surface connectivity.

\subsection{Topology in membrane phenomenology}

A range of experimental techniques have been applied in the characterization of the phase behaviour and morphologies of lipid-water systems. The existence of the lamellar bilayer phases and a number of bilayer phases with bicontinous structures exhibiting cubic symmetries have been complimented and confirmed with NMR [ ??] and freeze-fracture electron microscopy [ ??]. In the more diluted regimes of the phase diagram the characterization of the phases is complicated due to the absence of long range order in the phases and coexistence of a large number of bilayer structures. Direct visualization by microscope tecniques probably gives the best insight in the nature of the phases in this regime [??, ??].

A considerable effort has been directed toward characterization of the phases in terms of surface geometries [??]. The multi-lamellar and cubic phases have been characterized in terms of infinite periodic minimal surfaces (IPMS), e.g. the surfaces having zero mean curvatures and separating the ambient space into periodic subspaces. An IPMS has thus an associated point group symmetry and characteristic dimensions of its unit cell. The topology of an IPMS can be very complicated, e.g. represented by the number of genus per unit cell. Properties of minimal surfaces can be derived from complex analysis through their representation by Weierstrass-polynomials. However, IPMS is still not a fully determined group of surfaces, and the non-periodic minimal surfaces with non-trivial topology has only recently been explored. It is thus evident that surfaces are difficult to treat in a statistical mechanical frame if they are assumed to be minimal. Another difficulty in dealing with minimal surfaces in membrane physics is that a physical principle, which dictates the crystalline properties of the IPMS, is not known. No packing condition or internal symmetri property of the constituents can guide us, as in the case of molecular crystals. With these difficulties we find that minimal surfaces at present do not provide a good starting point for the description of membranes undergoing phase transitions involving topology. We shall in this section restrict ourselves to closed membranes, which actually never can be described as minimal surfaces in $R^{3}$.

Our original motivation for studying the differential geometrical model of membranes has been to extend the previously found solutions to simple non-trivial topological aspects, but it turned out that our solutions would predict much more complex topological structures than first anticipated. One might therefore ask if such topological complexity, i.e. a large conglomerate of handles and tubuli, has ever been observed in biological membranes. In figure 1 examples from intra-cellular membrane structures are shown, e.g. the Golgi apparatus, the Endoplasmic Reticulum, etc., where complicated topological structures of tunnels and pipes are interwoven in each other. Such structures are difficult to observe experimentally but can within a certain accuracy be derived from e.g. x-ray diffraction data. 


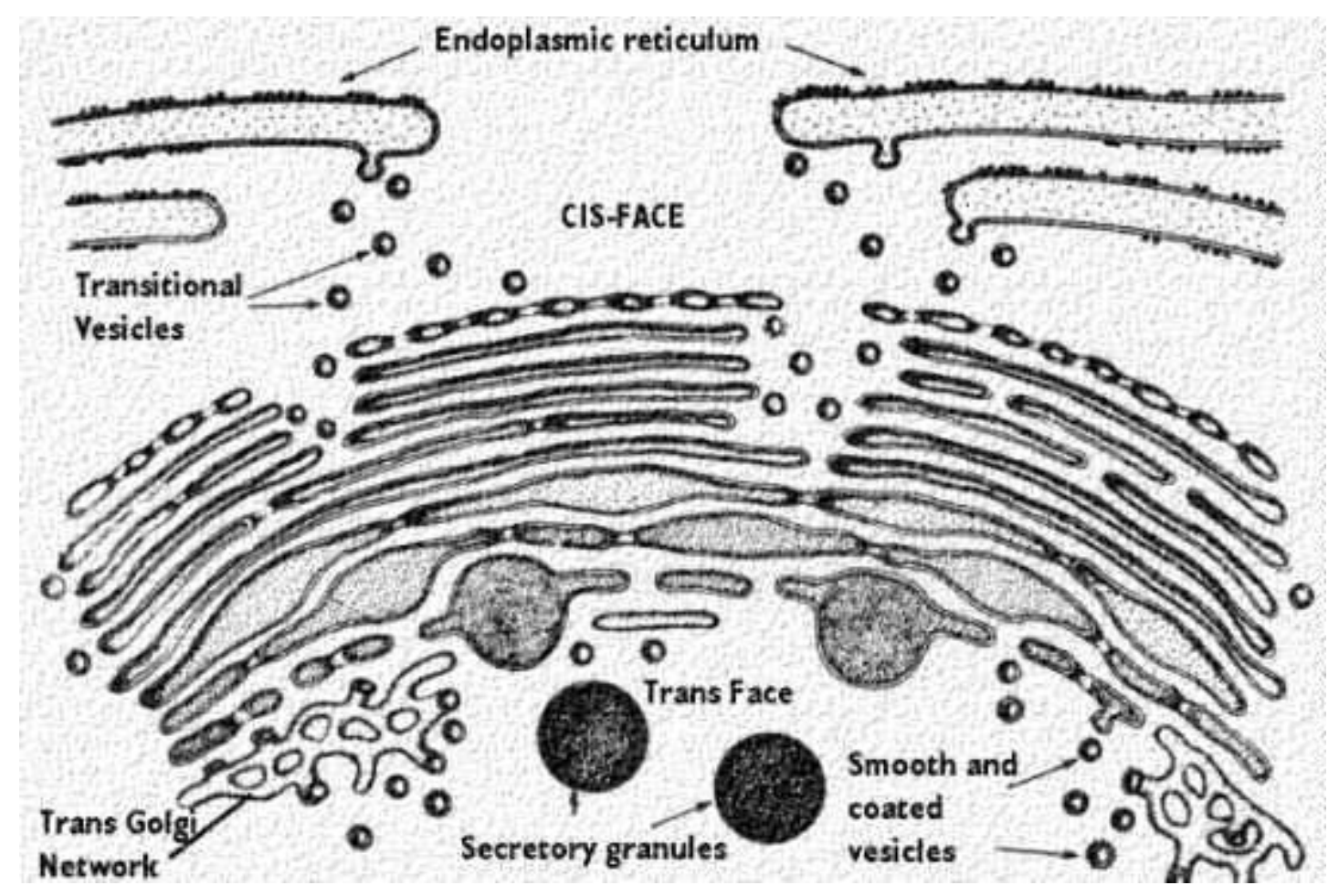

Figure 1: Schematic picture of a typical part of the endoplasmatic reticulum in animal cells[ ??].

\subsection{Model}

In this subsection the Canham-Helfrich model of membrane elasticity will be briefly described. This model considers only fluid membranes which at length-scales much larger than the molecular distances and the bilayer thickness can be modeled as a mathematical surface without any internal structure. The lipid bi-layers exhibit a number of low-temperature solid-like phases with in-plane order of the lipid molecules, but they do no demonstrate the deluge of large-scale structural transitions displayed by the fluid membranes. The model is based on the following Hamiltonian that takes the form

$$
\mathcal{H}=\mu \int d A+\frac{\kappa}{2} \int d A\left(\frac{1}{r_{1}}+\frac{1}{r_{2}}-\frac{2}{r_{0}}\right)^{2}+\bar{\kappa} \int d A \frac{1}{r_{1} r_{2}},
$$

where the integrations are performed over the surface area, $d A=d x_{1} d x_{2}, x_{1}, x_{2}$ being the embedded coordinates of the membrane surface. Here $r_{1}$ and $r_{2}$ are the local principal curvatures of the surface and $r_{0}$ is the spontaneous curvature, which can arise in bi-layers with an intrinsic asymmetry between the mono-layers of the bilayer. In this work we consider full symmetry between the two bilayer halves, which is the case when the bilayer is composed of a single molecular constituent. The notion of spontaneous curvature will thus be omitted in the following. The mean curvature $\frac{1}{r_{1}}+\frac{1}{r_{2}}$ and the gaussian curvature $\frac{1}{r_{1} r_{2}}$ are surface invariants, i.e. independent of the chosen parametrisation of the surface. The surface tension $\mu$, which couples to the surface area, must be considered as a chemical potential for the lipids in the membrane due to the fixed cross-sectional areas of the lipids. In most thermodynamic problems involving interfaces the chemical potential controls the interface.

When all the lipids in the lipid-water mixture form bilayer structures with a fixed cross-sectional area per lipid molecule, $\mu$ is negligible since the free lipid molecules are insoluble in water. $\mu$ may be non-negligible in realistic cases when a lipid bilayer phase is coexisting with a non-bilayer phase, when different bilayer phases are coexisting or in the case of black films. Other terms may be included in Eq. (1) . If the membrane has boundaries a line tension term

$$
\mu_{L} \int_{\text {boundary }} d \ell
$$

must be added. However the line tension $\mu_{L}$ is so large that even the presence of small boundaries are 
suppressed for free membranes. Boundaries can occur if the membrane can be attached to hydrophobic or hydrophillic elements of the experimental setup. Here, we do not consider these cases and just assume that the membranes are without boundaries. Furthermore anharmonic terms are neglegted in Eq.(1). The model parameters $\kappa$ and $\bar{\kappa}$ are difficult to obtain experimentally. However, some concensus have been reached regarding the value of $\kappa$ for artificial membranes.

\subsection{The Willmore functional}

In this subsection we discuss some results from the mathematical literature concerning the properties of the functional which appears as the second term in Eq.(1), the Willmore functional. The Willmore functional is written as

$$
W(\Sigma)=\frac{1}{2} \int_{\Sigma} H^{2} d A
$$

where $H=\frac{1}{r_{1}}+\frac{1}{r_{2}}$ is the mean curvature and $d A$ is the area element of a surface $\Sigma$. Here $\Sigma$ is any compact surface in $\mathbf{R}^{3}$ and we assume that it has no boundaries and no self-intersections. The functional $W$ is invariant under conformal mappings of the ambient 3 -space. Thus, if $\tilde{\Sigma}$ is the image of $\Sigma$ under a Möbius transformation (an isometry, a scaling or an inversion in a sphere with center not in $\Sigma$ ), then $W(\tilde{\Sigma})=W(\Sigma)$ [??]. Recently, considerable effort have been directed toward a solution of the Willmore problem for surfaces of any genus (concerning the infimum of $W$ and the related variational problem). A few results relevant for our purpose will be given.

Following L. Simon [??] we write $\beta_{g}=\inf W(\Sigma)$, where the infimum is taken over compact genus $\mathrm{g}$ surfaces without self-intersections. The following inequality is fundamental:

$$
8 \pi \leq \beta_{g}<16 \pi
$$

Equality holds on the left if and only if $g=0$ and $\Sigma$ is a round sphere [??]. The right hand side inequality was observed independently by U. Pinkall and R. Kusner, see [??]. Simon then showed [ ??], that if we consider $e_{g}=\beta_{g}-8 \pi$, then

$$
e_{g} \leq \sum_{j=1}^{q} e_{\ell_{j}}
$$

for any integers $q \geq 2$ and $\ell_{1}, \ldots, \ell_{q}$ with $\sum_{j=1}^{q} \ell_{j}=g$. Further he proved the existence of $W$-minimizers in the following sense: For any genus $g$ there exists a genus $g$ surface $\Sigma$ with $W(\Sigma)=\beta_{g}$, unless equality holds in Eq. (5) in which case there exists a sequence $\Sigma_{k}$ of genus g surfaces and a genus $g_{\circ}$ surface $\Sigma_{\circ}$ (with $g_{\circ} \leq g$ ) such that $W\left(\Sigma_{k}\right) \rightarrow W\left(\Sigma_{\circ}\right)=\beta_{g_{\circ}}$ for $k \rightarrow \infty$. Thus for a given surface the minimization of $\mathrm{W}$ may cause a drop in genus number. However, it is not known whether equality actually can occur in Eq. (5).

\subsection{Thermodynamics of surface topology}

Going back to the original Hamiltonian Eq.(1) we have in the last chapter given bounds on the second term, the Willmore functional. The third term is easily evaluated by using the Gauss-Bonnet theorem:

$$
\int_{\Sigma} d A \frac{1}{r_{1} r_{2}}=2 \pi \chi
$$

When the surface is without boundaries the Euler characteristic $\chi$ is simply related to the genus number by $\chi=2 \pi(2-2 g)$.

From the previous section it is clear that the Willmore functional restricted to compact embedded surfaces $\Sigma$ without boundaries is related to $\mathcal{H}: W(\Sigma)=\frac{1}{\kappa}(\mathcal{H}-\bar{\kappa} \chi(\Sigma))$. In particular $\inf _{g} \mathcal{H}\left(\Sigma_{g}\right)=$ $\kappa \inf _{g} W\left(\Sigma_{g}\right)-4 \pi \bar{\kappa}(1-g)$, where $\inf _{g} W$ represents the infimum of $W\left(\Sigma_{g}\right)$ for all boundary-less compact, embedded surfaces $\Sigma_{g}$ with genus $g$.

The next step is to sum up the contribution of the Willmore functional from each topological genus class. We shall be taking the extremum of the Willmore functional from each genus class. It's measure contributes to a number summed up to either almost 0 or to a finite number that could become infinite 
dependent on the degeneracy of the extremum. The sum would be correspondingly 0 or infinite. Hence, in order to obtain a meaningful result of the genus sum we shall assume each Willmore contribution in each class to be finite or that there is a finite degeneracy of the functional. We have reasons to believe that the Willmore functional is defined up to homotopy in finite exemplar. The assumption of finiteness is also rendered probable by computer simulation where surfaces can be triangularized and thus make a computer estimate of the Willmore functional possible.

We are now in the position to set up the partition function for a single closed membrane made out of A lipids. Note, by introduction of a fixed membrane area we break the conformal invariance explictly.

$$
\begin{aligned}
Z(A) & =\sum_{g=0}^{G_{A}} \operatorname{Tr}_{g}(\exp (-\beta \mathcal{H})) \\
& =\sum_{g=0}^{G_{A}} \exp (-4 \pi \bar{\kappa}(1-g)) \operatorname{Tr}_{g}\left(\exp \left(-\beta \frac{\kappa}{2} W\right)\right)
\end{aligned}
$$

The trace $\operatorname{Tr}_{g}$ represents the summation of all physical distinct surfaces $\Sigma_{g}$ with fixed area corresponding to $A$. Surfaces which are identical apart from a reparametrisation of the surface are not physically distinct. $G_{A}$ represents a cut-off in the number of genus for a membrane of size $A . G_{A}$ exists if the diameter and the sectional curvatures have upper bounds. This is indeed the case for a closed membrane due limited number of lipids $A$ involved and material parameters determined by size and physical properties of the molecular constituents. A first approximation to $Z(A)$ can be obtained by restricting $\operatorname{Tr}_{g}$ to surfaces which realize the minimum of $W$. This is - according the previous considerations - the case for one surface for each $g$. The structure of this surface is not known for general $g$ at present, which makes it impossible to go beyond this simple thermodymanical level of description. We will further reduce the number of degrees of freedom and assume that the membrane is motionally hindered, so $g$ is the only available degree of freedom and the minima of $W(\Sigma)$ are non-degenerate up to homotopy.

case 1.:

$$
Z(A) \approx Z^{\mathrm{SP}}(A)=\sum_{g=0}^{G_{A}} \exp (-4 \pi \beta \bar{\kappa}(1-g)) \exp \left(-\beta \frac{\kappa}{2} \inf _{g}(W)\right)
$$

By use of Eq.(4) and Eq.(7)

$$
Z_{1}(A) \leq Z^{\mathrm{SP}}(A) \leq Z_{2}(A)
$$

where

$$
Z_{c}(A)=\exp (-4 \pi \beta(2 \kappa+\bar{\kappa}))+\exp (-8 \pi \beta c \kappa) \frac{1-\exp \left(4 \pi \bar{\kappa} \beta G_{A}\right)}{1-\exp (4 \pi \beta \bar{\kappa})} \quad c=1,2
$$

For $G_{A} \rightarrow \infty, Z_{c}$ and $Z^{S P}$ are analytic for $\bar{\kappa}<0$, and $Z_{c}$ is solely controlled by the second term in Eq.(9) for $\bar{\kappa} \rightarrow 0^{-}$. From Eq.(8) it is evident that $Z^{S P}$ is also governed by a singularity of this nature as $\bar{\kappa} \rightarrow 0^{-}$. $Z_{c}$ can thus be considered as a good approximation for $Z^{S P}$ under these conditions. Note that $Z_{1}$ in this limit is essentially the partition function for a quantum harmonic oscillator. However, $1 \ll G_{A}<\infty$ is the regime of interest in the description of the physical system. Here $Z_{c}$ and $Z^{S P}$ are analytic for $\bar{\kappa} \neq 0$. The inequality in Eq.(8) holds term by term in an expansion of $Z_{c}$ and $Z^{S P}$ in $\exp (4 \pi \bar{\kappa}(1-g))$ like Eq.(7). It is then trivial to show that the inequality holds term by term in an expansion in $\bar{\kappa}$. The expansion coefficients are thermal expectation values which will be considered in the following.

The free energy derived from $Z_{c}, F_{c}=-\beta^{-1} \ln \left(Z_{c}(A)\right)$, and its thermal behaviour may be analyzed. The obvious orderparameter in this problem is the averaged genus $\langle g\rangle$ or the average Eulercharacteristics $<\chi>=4 \pi(1-<g>)$.

$$
\begin{aligned}
<\chi> & =-\frac{\partial F_{c}}{\partial(\beta \bar{\kappa})} \\
& =\beta^{-1} \frac{1}{Z_{c}(A)} \frac{\partial Z_{c}(A)}{\partial(\beta \bar{\kappa})}
\end{aligned}
$$




$$
=4 \pi \beta^{-1} \frac{1}{Z(A)}\left(-\frac{a}{x}+b \frac{-G_{A} x^{G_{A}}+\left(G_{A}-1\right) x^{G_{A}+1}+x}{(1-x)^{2}}\right)
$$

We have here used the notation $x=\exp (4 \pi \beta \bar{\kappa}), a=\exp (-8 \pi \kappa \beta)$ and $b=\exp (-8 \pi \kappa \beta c)$. The fluctuations in $\langle\chi\rangle$ can be expressed as

$$
\begin{aligned}
\sigma(\chi) & =<\chi^{2}>-<\chi>^{2} \\
& =-\frac{\partial^{2} F_{c}}{\partial(\beta \bar{\kappa})^{2}} \\
& =\beta^{-2}\left(\frac{1}{Z_{c}(A)^{2}} \frac{\partial^{2} Z_{c}(A)}{\partial^{2}(\beta \bar{\kappa})}-\left(\frac{1}{Z_{c}(A)} \frac{\partial Z_{c}(A)}{\partial(\beta \bar{\kappa})}\right)^{2}\right)
\end{aligned}
$$

$\langle\chi\rangle$ display a sudden, but continous change at $\bar{\kappa}=0$ from $\langle\chi\rangle \approx-4 \pi$ corresponding to a sphere for $\bar{\kappa}<0$ to $\left\langle\chi>\approx-4 \pi G_{A}\right.$ for $\bar{\kappa}>0$, see fig. 2. This transition is governed by strong fluctuations in $g$, which is manifested by a peak in $\sigma(\chi)\left(\approx\left(2 \pi G_{A}\right)^{2}\right)$ at $\bar{\kappa}=0$.

Case 2.

The simplest extension of case 1 is to consider a collection of immobile closed membranes which only interact through exchange of lipids giving rise to a vesicle size distribution. The partition function thus takes the form:

$$
Z=\sum_{N_{A}} \frac{e^{-\nu \sum_{A} N_{A} A}}{\prod_{A}\left(N_{A} A\right) !} \prod_{A} Z(A)^{N_{A}}
$$

where the sum runs over all possible vesicle size distributions $\left\{N_{A}\right\}$. The total amount of lipid in the system is controlled by the chemical potential $\nu$. A has a lower cut-off which is determined by the molecular details [??].

Case 3.

This case represents a collection of closed membranes, which can exchange lipids and are free to move in space. Only the translational degrees of freedom will be taken into account, because handling of the rotational degrees of freedom require detailed information about the structures of the Willmore surfaces for all $g$, which is not available.

In figure 2 we summarise these thermodynamical results by showing the various phases of case 1,2 and 3 in phase diagrams with the parameters $\kappa$ and $\bar{\kappa}$, the coefficients of the bending rigidity and the Gaussian curvature respectively. A phase transition line along $\bar{\kappa}=0$ is separating a topological phase, in the upper region of the diagram, with infinitely many handles (high genus number $g$, case 1) and a phase $(g=0)$ with many vesicles, case 2 and 3 , in the lower region of the phase diagram. This region is furthermore divided by a phase transition line, approximately along $\kappa=-2 \bar{\kappa}$, into a phase containing many smaller vesicles and a phase with one single large vesicle. In figure 3 we have focused on the case 2,3 and shown the average order parameter $\langle-1 / 2 \chi\rangle$ and the fluctuation of $\chi$ as a function of $\bar{\kappa}$ and furthermore the size distribution of vesicles at different $\bar{\kappa}$ and fixed $\kappa$. These distributions are exactly what we expect of the theory in case 3 .

\subsection{Discussion and Conclusion}

In the previous section the biophysical problem on the thermodynamic properties of closed membranes is related to a variational problem on Willmore surfaces. This mathematical problem is still unresolved but the results obtained so far gives sufficient information to provide valuable results on the phase behaviour of membranes. Three simple cases was considered: case 1 an ensemble of independent and motionally restricted membranes. In this case the system display an abrupt, continous change in $\langle g\rangle$ at $\bar{\kappa}=0$. Although it is accompanied by strong fluctuations in $\langle g\rangle$, the transition is neither 1. order or 2 . order, but rather $\infty$ order in the sense that $\frac{\partial^{n} F}{\partial(\beta \bar{\kappa})} \propto\left(G_{A}\right)^{n} \rightarrow \infty$ for $n \rightarrow \infty$ at $\bar{\kappa}=0$. In an ensemle of weakly interacting membranes this may be changed to a 1 . order or a 2 . order transition.

The principles behind the interplay between three dimensional structure and the structural transitions of proteins and their biological function are to a large extent understood. A similar relationship for the biological membranes is still considered at a hypothetical level [??]. Whether the extended lipid polymorphism has any significance in biological system is still unclear. 

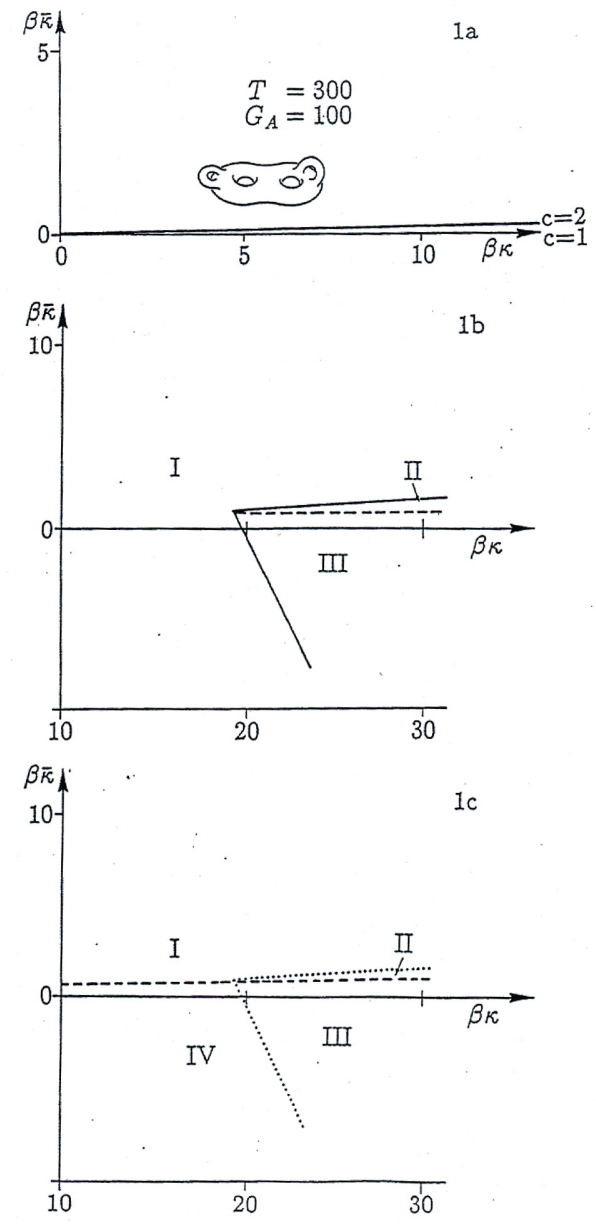

Figure 2: The figure shows 3 phase diagrams in the parametres $\beta \kappa$ and $\beta \bar{\kappa}$ of the partition function for the lipid system. Fig. 1a, $1 \mathrm{~b}$ and $1 \mathrm{c}$ correspond respectively to case 1, case 2 and case 3.

Of great interest in this contribution are the topological properties of membranes. It is observed [ ??] that large-scale structures of lipid membranes exhibit great topological complexity which is realized in biological membranes, for example in the Golgi-apparatus, see figure 1, inside the cell. Here are lots of handles and tubuli connecting different compartments between layers. The purpose of this complex topological structure is a need for filtration of proteins in the cellular liquid. An interesting question is then how one could explain dynamically the formation of handles or tunnel structure and division into small vesicles. Such an explanation requires a theory of topology dynamics.

The topological complexity appearing in biological membrane structures, e.g. the intra-cellular Golgi apparatus, can largely be explaned by the phase diagram of figures 2 and 3, where an "explosion" of handles and tubuli occur and only limited by material constraints such as a finite lipid size. The phase transition around $\kappa=-2 \bar{\kappa}$, between single vesicle structure and many small vesicles can also be observed in experiments [ ??]. It seems crucial for cell growth, in an early state of life, that the membrane structure is balancing between the two situations as if life tends to stay in non-equilibrium.

\section{SECTION II: The topology of 2-dimensional protein surfaces}

Previously we considered smooth, biomolecular systems at the typical size of hundreds to thousands of nanometers. We shall now zoom down to particular biomolecules, proteins typically of the size of five to ten nanometers, that also have a well-defined differential geometric structure that explains aspects of their biofunctionality and material science features. 

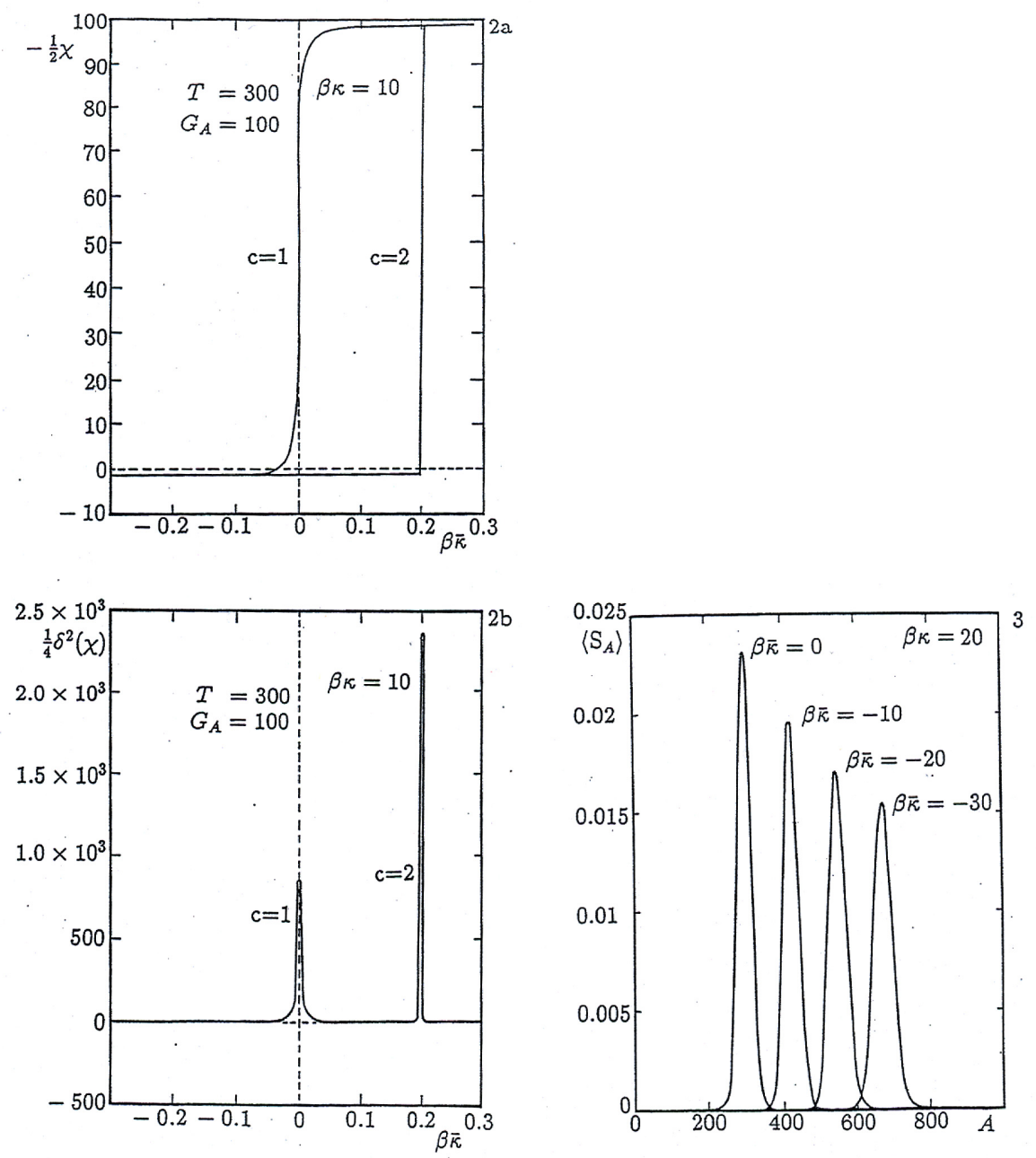

Figure 3: A) Figure 3 shows profile of genus g, or actually $-1 / 2 \chi$, along line $\kappa=3.0$ which has discontinuity around $g=0$ Profile is for both $c=1$ and $c=2$ Left-upper figure 2a shows average order parameter $\langle-1 / 2 \chi\rangle$ as a function of $\bar{\kappa}$ while Left-lower figure $2 \mathrm{~b}$ shows the fluctuations of $\chi$ as function of $\bar{\kappa}$. B) Right figure (3) shows several size distributions of vesicles with different values of $\bar{\kappa}$ and fixed $\mu \kappa$

\subsection{Construction of surfaces for proteins from their coordinates}

It is not a trivial task to construct a smooth surface from a 3-dimensional protein structure, especially if the surface has to contain all the protein backbone. In order to see that, one should visualize the protein as a cluster of atoms connected to each other by an unbranched chain that winds in and out of the center of the cluster.

In X-ray crystallography as well as in NMR the problem is to construct the 3-dimensional protein structure from spectroscopic data that provide distances between some of the atoms in the protein. Such construction is an expansion of 2-dimensional information in the form of distance maps to 3-dimensional structural information which requires large processing capacity and some extra chiral information or chain topology. Nevertheless it has been one of the most important processing tasks of this century since it gave science all the known 3-dimensional structures of bio-molecules, as well as other molecules, gathered together in huge databases. There has now been devised various short-cuts to the problem of $2 \mathrm{~d}$ to $3 \mathrm{~d}$ expansion [??, ??].

Before discussing how to construct surfaces one should decide what kind of surface one wants. The most obvious one is the "outer" surface or also called the solvent accessible surface for a protein. 


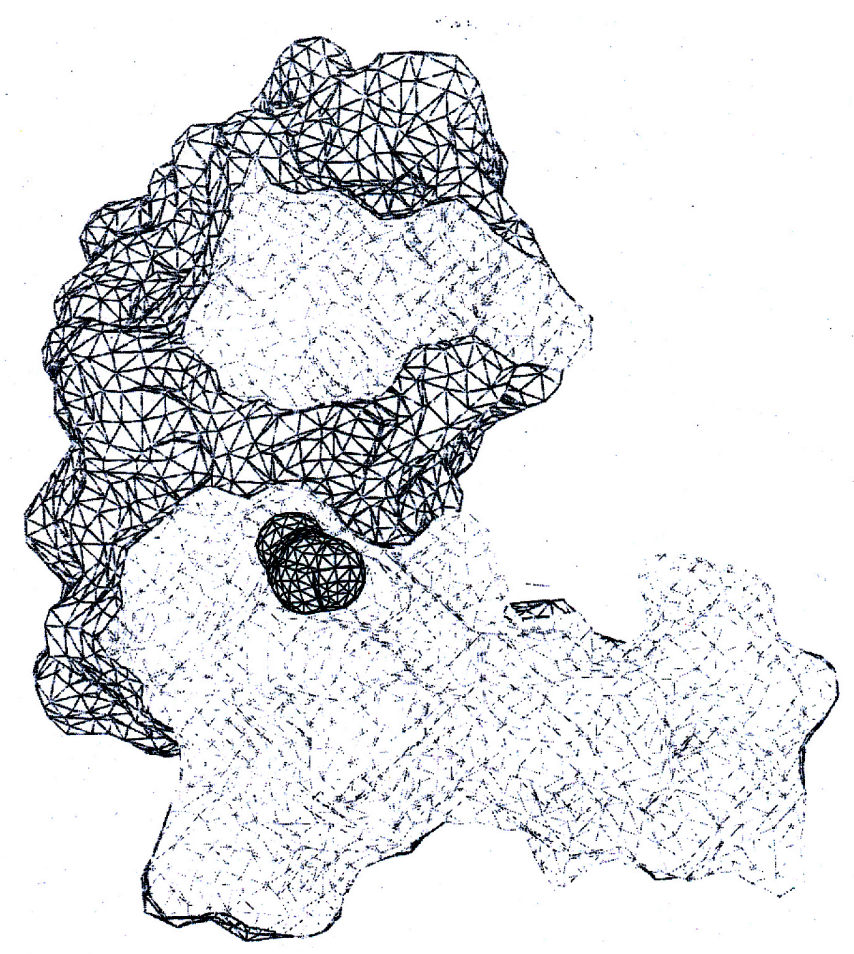

Figure 4: A construction of the solvent accessible surface for the protein, lysozyme. The darker color represents the outer surface while the lighter color represents the inner surface. A ligand is embedded in the surface.

This solvent accessible surface is most commonly obtained by rolling a ball on the protein structure given by coordinates thereby obtaining a subset of the protein coordinate set that could be in contact to a solvent with the size of the rolling ball. This is of course all done in a simulation by a computer. The ball size can then be varied in order to get into grooves and cavities or, oppositely, in order to avoid them by making it smaller, respectively, larger. There are many standard programs for this procedure. In this review we have produced some pictures with the help of the Connolly packet [??]. Once the outer solvent accessible surface has been made one can then construct (a triangulation of) the electrostatic potential surface or the Van der Vaal contact surface as shown in Fig. 4, and hence find the curvature tensors.

\subsection{Differential geometry of the protein surface}

We start again by considering the constructed 2-dimensional protein surface embedded in a 3 -dimensional Euclidean space. We shall only be considering embedded surfaces with no intersections. The surface $\{\bar{x}(\sigma)\}$, which can be described by a parametrization $\sigma\left(\sigma_{1}, \sigma_{2}\right)$, is completely determined, up to Euclidean transformations, by the two tensors, the metrical tensor $g_{i j}$ and the extrinsic curvature tensor (the second fundamental form) $K_{i j}$. The induced metric tensor $g_{i j}$ is of course:

$$
g_{i j}=\partial_{i} \bar{x} \partial_{j} \bar{x}
$$

where $\partial_{i}=\frac{\partial}{\partial \sigma_{i}}$. The extrinsic curvature tensor, $K_{i j}$ is:

$$
K_{i j}=\bar{n} \partial_{i} \partial_{j} \bar{x}
$$

where $\bar{n}$ is the unit normal perpendicular to the tangential surface, $\bar{n} \cdot \partial_{i} \bar{x}=0$ for $i=1,2 . \bar{x}, g_{i j}$ and $K_{i j}$ are related through the Gauss-Weingarten equations:

$$
\partial_{i} \partial_{j} \bar{x}=\Gamma_{i j}^{\alpha} \partial_{\alpha} \bar{x}+K_{i j}^{\alpha} \bar{n} \quad \text { where } \quad \Gamma_{i j}^{\alpha} g_{\alpha \kappa}=\partial_{i} \partial_{j} \bar{x} \partial_{\kappa} \bar{x}
$$

If we want to construct a surface model with an energy function it must be invariant with respect to changes of the coordinate system. Therefore the terms of an eventual energy function must contain 


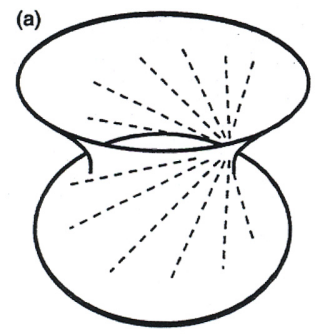

(b)

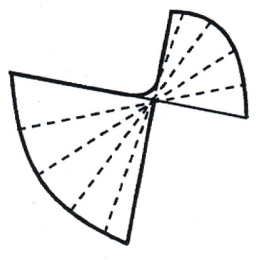

Figure 5: A picture of the two most well-known minimal surfaces (shown cut-out with boundary), (a) the catenoid and (b) the helicoid. From ref. [??].

surface invariants and they can only be constructed from the the curvature, metrical and torsional tensors up to third order in the derivatives. The first invariants to be included in an expansion of the free energy of the surface are:

$$
1, \quad g^{i j} K_{i j} \text { and } K_{j}^{i} K_{j}^{i},
$$

where $K_{i}^{i}$ is the mean (exterior) curvature and $G=K_{i}^{i} K_{j}^{j}-K_{i}^{j} K_{j}^{i}$ is the Gaussian curvature. These quantities can also be expressed in term of the local principal radii of curvature $r_{1}$ and $r_{2}$ :

$$
K_{i}^{i}=\left(\frac{1}{r_{1}}+\frac{1}{r_{2}}\right), \quad G=\frac{1}{r_{1} r_{2}}
$$

The next invariants will contain 2-forms of higher order derivatives.

One can now write the Hamiltonian function as in section 1 but this time for the protein surface that contains these surface invariants:

$$
\begin{aligned}
H & =\mu \int d^{2} \sigma \sqrt{\operatorname{det}(g)}+k_{1} \int d^{2} \sigma \sqrt{\operatorname{det}(g)}\left(K_{i}^{i}\right)^{2}+k_{2} \int d^{2} \sigma \sqrt{\operatorname{det}(g)} G \\
& +\quad \text {.higher order terms } .
\end{aligned}
$$

\subsection{Minimal surfaces of proteins}

We shall in this chapter discuss the importance of minimal surfaces for protein functionality and especially enzymatic reaction kinetics. In the last chapter we saw how surfaces for proteins could be constructed from the set of atomic coordinates by triangularization. As it turns out several important proteins have a solvent accessible surface that resembles that of minimal surfaces and their enzymatic functioning is very dependent on that.

It is not surprising that protein surfaces often appear as minimal surfaces since, as e.g. in the case of beta-sheets, a network of hydrogen bonds is forcing the surface to maintain constant zero mean curvature such that a bend in one direction will cause an opposite bend in another direction. This can be understood by applying the Poisson equation that seek to maintain constant average density on the protein surface.

First, some words about minimal surfaces arising from infinite systems. Minimal surfaces have their mean curvature overall equal to zero: $K_{i}^{i}=0$. In general the minimal surfaces are infinite but they are often considered and drawn with a boundary, such as for the catenoid or helicoid, see figure 5 . One of the most famous minimal surfaces is the helicoid, which can be generated as a ruled surface. The simplest minimal surface is the plane. A minimal surface generally has a Weierstrass representation where the coordinates of a point in 3D Euclidean space on the surface is given by the function $\mathcal{F}$ of complex variables $\omega=\sigma+i \tau$ in the plane produced from the minimal surface by a conformal parametrization:

$$
x=\operatorname{Re} \int\left(1-\omega^{2}\right) \mathcal{F}(\omega) d \omega
$$



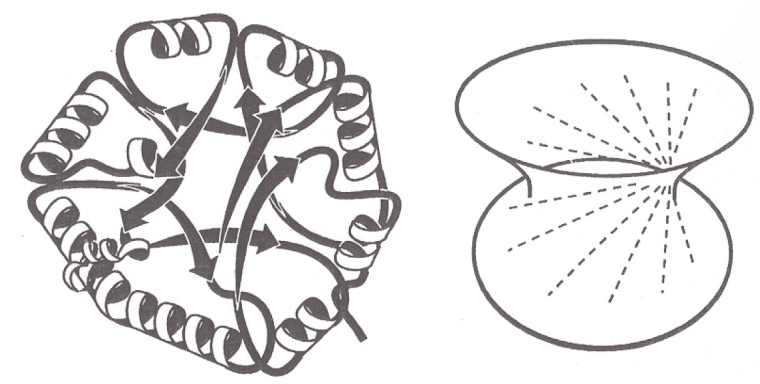

Figure 6: A constructed picture of the protein, Triose Phosphate Isomerase, schematically embedded into a catenoid surface [??]

$$
\begin{aligned}
& y=\operatorname{Im} \int\left(1+\omega^{2}\right) \mathcal{F}(\omega) d \omega \\
& z=\operatorname{Re} \int 2 \omega \mathcal{F}(\omega) d \omega
\end{aligned}
$$

\section{The surface geometry of protein reaction kinetics}

When looking at protein functionality in general, and especially for enzymatic reactions, it is not surprising that the geometry of the surface of the protein plays an important role. By the surface we of course again mean the solvent accessible surface, SAS. This dependence on the surface geometry is especially seen when the functionality is about binding to a substrate, antibody-antigen interaction, enzymatic hydrolysis of peptide bonds, charge transfer etc. We shall in the following consider the particular process of substrate (ligand) binding or ligand transferring and relate that to the minimal surface property.

\subsection{Minimal surfaces and protein-ligand interaction}

Coming back to protein surfaces there are obvious cases where the SAS of a protein seems to resemble a minimal surface, at least partly, and which seems to have profound reasons for the functioning of that particular protein. Such cases are often involving particular topological patterns of secondary structures. The word topology is here used in the meaning of a graph theoretical sense where the various secondary structure elements are connected to each other in a particular way. Typically these patterns consist of an alternating, symmetric occurrence of alpha helices and beta strands, $\alpha / \beta$. This is seen in the $\alpha / \beta$ barrel proteins, such as the triose phosphate isomerase where the beta strands, see figure 6 , intertwine the alpha helices and form a particular core structure with a barrel structure and which can be described by the center portion of the catenoid minimal surface. The open end of the catenoid is then supposed to contain the active site while the tunnel of the catenoid is formed by the hydrophobic residues of the beta strands which are tilted relative to the axis of the tunnel. The beta sheets are straight meaning that the normal curvature is zero and their relative twist is equal to the geodesic torsion. The value of the twist gives the magnitude of the Gaussian curvature, G. The less symmetrical $\alpha / \beta$ protein, the redox favodoxin, has similarly a beta sheet core that is well described by the helicoid minimal surface. Thus, we base our analysis of enzymatic processes on the curvature tensors K and G calculated from triangularization of the SAS.

We shall now, following the argument in the book of Hyde et al. [??], see how these minimal surfaces can be important for the enzymatic processes the two mentioned proteins are involved with. Most commonly the enzymatic processes of a protein involve hydrolysis of some sort or binding to a substrate, e.g. a particular ligand. In the latter case it is important for the understanding of the process and its effectiveness that one can explain how the diffusion of the ligand is steered through the protein rather than being a matter of "random encounter chemistry" which never would be able to explain the extremely high yield of protein catalysed reaction kinetics. The driving mechanism in the so-called "forced diffusion" of 
the catenoid modelled beta-barrel protein is the guiding gradient of the Gaussian curvature which attain its most negative value in the waist of the catenoid. Thus a ligand approaching the protein from infinity will be smoothly guided through the catenoid tunnel of the protein towards the waist and the active site. The minimal surface will guarantee smooth and swift diffusion of the ligand towards and through the protein. Indeed, the negative Gaussian curvature of any minimal surface locally speeds up the Brownian diffusion process in comparison with the corresponding diffusion on a flat surface, see [??]. However, a spherically cut off part of any minimal surface also has larger area than a similarly cut off part (disk) in the plane. It turns out, as a consequence of minimality, that the larger area balances precisely the faster Brownian motion, so that the Brownian motion mean exit times are exactly identical on the two domains: the sperically cut off part of the minimal surface and the disk, respectively. The specificity of the reaction is given by the particular combination of the geometry and side-chain composition of the ligand and protein. Concerning construction of minimal surfaces we can refer to [??] [??].

\subsection{Conclusion}

In section 2 we have analyzed constructed protein surfaces that can be triangularized in order to provide differential geometric information. Usually the protein folding problem is seen as a matter of going from 1 dimensional representations to 2 dimensional (secondary) structures and then going to the full 3 -dimensional (tertiary) structures. However, when going to 2-dimensional surface structures one can by means of minimal surfaces understand enzymatic activities.

\section{Acknowledgement}

We wish to thank the employees at department of physics at DTU and the group at Memphys of SDU for support. Especially Dr Gerhard Besold is acknowledged.

\section{References}

1. Luzzati V., Tardieu, A. (1974) Annu. Rev. Phys. Chem. 25. 79-92.

2. Israelachvili, D., Mitchell, J.,Ninham, (1977) Biochim. Biophys. Acta 470, 185-201.

3. Canham, P.B. (1970) J. Theoret. Biol. 26, 61-81; Helfrich, W. (1973) Z. Naturforsch. 28c, 693-703.

4. Deurling, H. J., Helfrich W. (1980) Biophys. J. 13, 941.

5. Helfrich W. (1978) Z. Naturforsch. 33a, 305 and Lipowsky R., Leibler S. (1986) Phys. Rev. Lett. $56,2541$.

6. Helfrich W. (1985) J. Physique 46, 1263

7. Peliti L., Leibler S. (1985) Phys. Rev. Lett. 56, 1690.

8. Y Kantor, M Kardar, DR Nelson (1988) Phys. Rev. Lett. 60, 238.

9. Lindblom G., Wennerström H. (1977) Biophys. Chem. 6, 167-171.

10. Gulik-Krzywicki T., Aggerbeck L. P., Larsson K. in Surfactants in Solutions (Mittel K. L., Lindman B., Eds.) Vol. 1 pp 237-257, Plenum, New York 1984.

11. Miller D. D., Bellore J. R., Evans D. F., Talmon Y., Ninham B. W. (1987) J. Phys. Chem. 91, 674-685.

12. Servus R. M. (1989) Chemistry and Physics of Lipids 50, 87-97.

13. Anderson S., Hyde S.T., Larsson K., Lidin S. (1988) Chem. Rev.,88, 221-242.

14. Weiner J. (1978) Indiana Univ. Math. J. 27, 19-35. 
15. Kusner R. (1989) Pacific J. Math. 138, 317-345.

16. Simon L. (1986) Proc. Cont. Math. Anal. Aust. Natl. Univ. 10, 187-216.

17. University of Texas Medical Branch: www.cytochemistry.net/Cell-biology/golgi.htm

18. Willmore T.J., Total Curvature in Riemanian Geometry, Chichester, Wiley (1982).

19. Cullis P.R., Hope M.J., de Kruijff B.,Verkleij A.J., Tilcock C.P.S., "Phospholipids and Cellular Regulation" (ed.: J.F.Kou) CRC Press, Boca Raton, Florida (1985) Vol. 1.

20. H. Bohr and J. H. Ipsen, in "Characterizing Complex Systems", Ed. H. Bohr, pp. 74-79, Singapore, World Scientific Publishing Co. (1989).

21. W. Helfrich, W. Harbich in Physics of Amphiphilic layers, (J. Meunier, D. Langevin, N. Boccara, Eds) pp 58, Springer, Berlin, (1987).

22. R. E. Goldstein and S. Leibler, Model for laminar phases of interacting lipid membranes, Phys. Rev. Lett., 61, 2213-2216, (1988).

23. B. J. Litman L. (1973) Biochemistry, Vol. 12, 2545, and F. Szoka and D. Papahadjopoulos (1980) Annual Review of Biophys. and Bioeng. Vol.9, 467.

24. J. Bohr, H. Bohr, S. Brunak, R. M. J. Cotterill, B. Lautrup and S. B. Petersen, J. Mol. Biol., Vol. 231, 861-871 (1993).

25. M. L. Connolly, "Molecular Surface Package Manual, Version 2.6, Menlo Park, CA (1994).

26. S. Hyde, S. Andersen, K. Larson Z. Blum, T. Landh, S. Ledin and B. W. Ninham, "The Language of Shape", Elsevier, Amsterdam (1997).

27. S. Andersen, S. T Hyde, K. Larson and S. Ledin, Chem. Rev., Vol. 88, 221 (1988).

28. H. A. Schwartz, in "Gesammelte Mathematische Abhandlungen", Springer, Berlin (1890).

29. S. Markvorsen and M. Min-Oo, "Global Riemannian Geometry: Curvature and Topology", Birkhuser Verlag, Basel - Boston - Berlin (2003).

30. T.H. Colding and W.P. Minicozzi II, PNAS, Vol. 103, 11106-11111 (2006). 